\title{
Sur deux catégories remarquables de fonctions de variable réelle.
}

\author{
Par \\ H. Looma n (Utrecht).
}

M. A. Denjoy ${ }^{1}$ ) a défini deux catégories de fonctions de variable réello, a savoir les fonctions approximativement continues et ¿ préponclérance de continuité d'une part, les dérivées approximativos et los nombres dérivés prépondérants (de fonctions continues) d'autre part, dont il a démontré, en appliquant la partie réciproque du théorèmo de $\mathrm{Bair} \theta^{2}$ ), qu'elles sont limites de fonctions continues.

Le but de cette Note est démontrer comment on peut former des suites de fonctions continues, tendant vers les fonctions en question; la seule propriété de ces fonctions que j'ai à appliquer est colle exprimée par leur définition.

\section{Les fonctions approximativement continues et les fonctions à prépondérance de continuité.}

1. Soit f $f^{\prime}(x)$ une fonction mesurable, définie pour $a<x<b$ et Anie sur une épaisseur pleine de $(a, b)$. Posons $a<x_{1}<x_{2}<b$, $0<\lambda<1$.

La borne supérieure stricte (et en même temps la plus grande) des nombres $y$, satisfaisant à

$$
\left.\operatorname{mes}_{x_{1}}^{x_{1}} E^{\prime} \mid f^{\prime}(x)<y\right] \leqslant \lambda\left(x_{8}-x_{1}\right)
$$

1) Sur ortaines olasses de fonotions de variable relle. C. K. t. 162, 1916. Sur les Jonctions deriveses sommables, Bull. Soc. Math. 1915, p. 165-186. Sur la totalisation des nombres dérinds non sommables. Ann. Ec. Norm. Sup. 1916. p. 198. Sur uns proprieté des fonotions dérivées. Enseignomont Mathématique. 1916.

2) c.-ri-ll. le théorème: toute fonction ponctuellement discontinue sur tout ennemble parfait est limito de fonctions continues. 
est alors un nombre fin $1 \Phi\left(x_{1}, x_{3}, \lambda\right) ; \Phi$ est done déterminée par les deux conditions:

$$
\begin{gathered}
\underset{x_{2}}{x_{1}} E\{f(x)<\Phi\} \leqslant \lambda\left(x_{2}-x_{1}\right) \\
\operatorname{mes}_{x_{1}}^{x_{1}} E\{f(x)<\Phi+\varepsilon\}>\lambda\left(x_{2}-x_{1}\right),
\end{gathered}
$$

si petit que soit $\varepsilon>0$.

De même, la borne inférieure stricte (et en même temps la plus petite) des nombres $z$, satisfaisant à

$$
\operatorname{mis}_{\mathbf{m}_{1}}^{x_{3}} L^{i}\{f(x) \leqslant z\} \geqslant \lambda\left(x_{2}-x_{1}\right)
$$

est un nombre fini $\varphi\left(x_{1}, x_{2}, \lambda\right) ; \varphi$ est déterminée par les deux conditions :

$$
\begin{gathered}
\operatorname{mes}_{x_{1}}^{x_{3}} E\{f(x) \leqslant \varphi\} \geqslant \lambda\left(x_{2}-x_{1}\right) \\
\operatorname{mes}_{2} E\left\{f(x) \leqslant \varphi-\varepsilon^{\prime}\right\}<\lambda\left(x_{2}-x_{1}\right)
\end{gathered}
$$

si petit que soit $\varepsilon^{\prime}>0$.

On a immédiatement:

$$
\operatorname{mes}_{1} E\{f(x) \leqslant \Phi\} \geqslant \lambda\left(x_{2}-x_{1}\right)
$$

done $\varphi \leqslant \Phi$. On peut avoir $\varphi<\Phi$.

Si $x_{1}, x_{2}$ sont fixes et $\lambda$ varie de 0 à $1, \varphi$ et $\Phi$ ne décroissent jamais De là, on déduit aisément, en appliquant la définition de $\varphi$ et $\Phi$, que l'ensemble des valeurs de $\lambda$, tels que $\varphi\left(x_{1}, x_{2}, \lambda\right)<$ $<\Phi\left(x_{1}, x_{2}, \lambda\right)$, est fini ou dénombrable ( $x_{3}$ et $x_{2}$ fixes).

2. Je vais démontrer que, si $\lambda$ est fixe, les fonctions $\Phi\left(x_{2}, x_{1}, \lambda\right)$ et $\varphi\left(x_{1}, x_{2}, \lambda\right)$ sont respectivement semi-continue supérieurement et semicontinue inférieurement par rapport au couple $\left(x_{1}, x_{2}\right)$.

En effet, si $\Phi\left(x_{1}, x_{2}, \lambda\right)$ n'était pas semi-continue supérieurement, il existerait un couple fixe $x_{1}, x_{2}$ et des couples $x_{1}^{\prime}, x_{2}^{\prime}$, tels que l'on aurait:

$$
\Phi\left(x_{1}^{\prime}, x_{2}^{\prime}, \lambda\right)>\Phi\left(x_{1}, x_{2}, \lambda\right)+\varepsilon
$$

où: $x_{1}=\lim x_{1}^{\prime}, x_{2}=\lim x_{2}^{\prime} ; \varepsilon>0$ indépendant de $x_{1}^{\prime}, x_{2}^{\prime}$.

D'où:

$$
\begin{aligned}
\operatorname{mes}_{x_{1}^{\prime}}^{x_{2}^{\prime}} E\left\{f(x)<\Phi\left(x_{1}, x_{2}, \lambda\right)+\varepsilon\right\} & \leqslant \operatorname{mes}_{x_{1}^{\prime}}^{x_{2}^{\prime}} E\left\{f(x)<\Phi\left(x_{1}^{\prime}, x_{2}^{\prime}, \lambda\right)\right\} \\
& \leqslant \lambda\left(x_{2}^{\prime}-x_{2}^{\prime}\right),
\end{aligned}
$$




\section{Sur les fonctions de variable réelle}

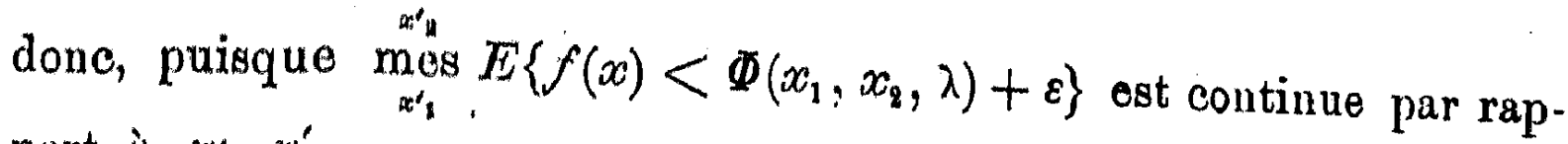
port is is $x_{1}^{\prime \prime}, x_{2}^{\prime}$,

$$
\operatorname{mes}_{\alpha_{1}}^{\alpha_{A}} \in L^{\prime}\left\{f^{\prime}(x)<\Phi\left(x_{1}, x_{2}, \lambda\right)+\varepsilon\right\} \leqslant \lambda\left(x_{2}-x_{1}\right),
$$

ce qui est en contradiction avec la définition de $\Phi$.

Ruisonnement analogue pour démontrer que $\varphi$ est semi continue inférieurement.

Donc, sur un segment $a_{1} b_{1}, \Phi\left(x_{1}, x_{2}, \lambda\right)$ et $\varphi\left(x_{1}, x_{2}, \lambda\right)$ sont borméos, puisque $\varphi$ est bornóe inférieurement, $\Phi$ est bornée supérieuroment, et $\phi \leqslant \phi$.

3. Posons $\left(0<\lambda_{1}<\lambda_{2}<1, x_{1}\right.$ et $x_{2}$ tixes $)$ :

$$
\psi\left(x_{1}, x_{2}, \lambda_{1}, \lambda_{2}\right)=\frac{1}{\lambda_{2}-\lambda_{1}} \int_{\gamma_{1}}^{\lambda_{9}} \Phi\left(x_{1}, x_{2}, \lambda\right) d \lambda,
$$

et puique $\varphi$ et $\Phi$ sunt des fonctions non décroissant de $\lambda$ :

$$
\Phi\left(x_{1}, x_{2}, \lambda_{1}\right) \leqslant \psi\left(x_{1}, x_{2}, \lambda_{1}, \lambda_{2}\right) \leqslant \varphi\left(x_{1}, x_{2}, \lambda_{2}\right) .
$$

Je dis que, $\psi\left(x_{1}, x_{2}, \lambda_{1}, \lambda_{2}\right)$ est une fonction continue de $\left(x_{1}, x_{2}\right)$.

Fin effet, soit $\lambda$ un nombre compris entre $\lambda_{1}$ et $\lambda_{2}$, et supposons que les 'nombres $x_{1}^{\prime}, x_{2}^{\prime}$ tendent respectivement vers $x_{1}$ et $x_{2}$. D'après $\& 2$, on a:

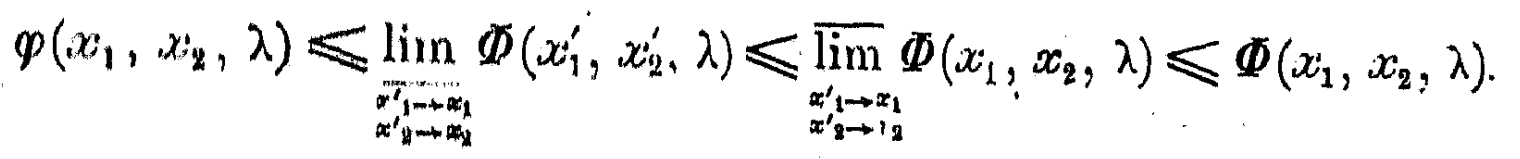

Donc, sauf pout-être pour une infinité dénombrable de valeurs de $\lambda_{i} \Phi\left(x_{1}^{\prime}, x_{2}^{\prime}, \lambda\right)$ (et d'ailleurs aussi $\varphi\left(x_{1}^{\prime}, x_{2}^{\prime}, \lambda\right)$ ) tend vers $\varphi\left(x_{1}, x_{2}, \lambda\right)=$ $=\Phi\left(x_{1}, x_{2}, \lambda\right)$.

De plus, $P$ et $\Phi$ sont bornées (pour $\lambda_{1}<\lambda<\lambda_{g}$ ), puisque un a toujours:

$$
\varphi\left(x_{1}^{\prime}, x_{2}^{\prime}, \lambda_{1}\right) \leqslant \varphi\left(x_{1}^{\prime}, x_{2}^{\prime}, \lambda\right) \leqslant \Phi\left(x_{1}^{\prime}, x_{2}^{\prime}, \lambda\right) \leqslant \Phi\left(x_{1}^{\prime}, x_{2}^{\prime}, \lambda_{2}\right)
$$

et $\varphi\left(x_{1}^{\prime}, x_{2}^{\prime}, \lambda\right), \Phi\left(x_{1}^{\prime}, x_{2}^{\prime}, \lambda\right)$ sont bornées.

Donc, d'après le théorème de M. Lebesgue sur le passage a la limite rous lo signe $f$ pour les fonctions bornées, on a:

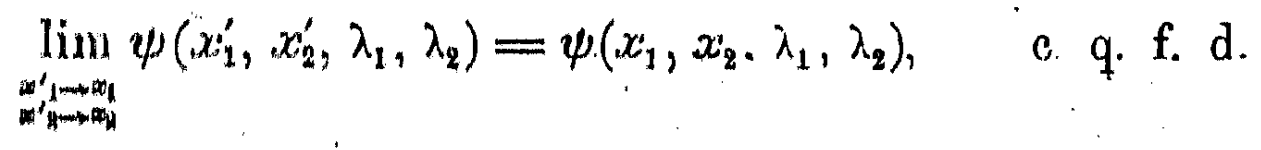


4. Pour toute fonction mesurable $\left.f(x)^{1}\right)$, finie sur une épaisseur pleine, nous pouvons donc déterminer une suite de fonctious continues: $f_{x}(x)=\psi\left(x, x+\frac{1}{n}, \lambda_{1}, \lambda_{2}\right)$.

Nous allons montrer que $f(x)$ tend vers $f(x)$, si $f(x)$ est approximativement continue $\mathrm{du}$ côté droit, c.-à.-d. si en tout point $x_{0}$, les fonctions

$$
\operatorname{mes}_{a}^{x} E\left\{f(x)<f\left(x_{0}\right)+\varepsilon\right\} \text { et } \underset{\omega}{x} E\left\{f(x)>f\left(x_{0}\right)-\varepsilon\right\}
$$

possèdent une dérivée à droite égale à un, si petit que soit $\varepsilon>0$.

Dunc, si $\varepsilon$ et $\varepsilon^{\prime}$ sont deux nombres positifs arbitrairement petits ( $\varepsilon^{\prime}$ inférieur à $\lambda_{1}$ et $\left.1-\lambda_{2}\right)$, il existe un nombre $N\left(x_{0}, \varepsilon, \varepsilon^{\prime}\right)$ tel que l'inégalité $n>N$ entraîne:

$$
\begin{aligned}
& \operatorname{mes}_{x_{0}}^{x_{0}+\frac{1}{n}} E\left\{f(x)>f\left(x_{0}\right)-\varepsilon\right\}>\left(1-\varepsilon^{\prime}\right) \frac{1}{n}, \\
& \operatorname{mes}_{x_{0}}+\frac{1}{n} E\left\{f(x)<f\left(x_{0}\right)+\varepsilon\right\}>\left(1-\varepsilon^{\prime}\right) \frac{1}{n} .
\end{aligned}
$$

On a aussi d'après les définitions de $\varphi$ et de $\Phi$ :

$$
\begin{array}{rl}
\operatorname{mes}_{x_{0}} & E\left\{f(x)>\varphi\left(x_{1}, x_{2}, \lambda_{1}\right)\right\} \leqslant\left(1-\lambda_{1}\right) \frac{1}{n}<\left(1-\varepsilon^{\prime}\right) \frac{1}{n} \\
& \operatorname{mes}_{x_{0}} E\left\{\frac{1}{n}\right.
\end{array}
$$

Done

d'où:

$$
f\left(x_{0}\right)-\varepsilon<\varphi\left(x_{1}, x_{8}, \lambda_{1}\right) \text { et } f\left(x_{0}\right)+\varepsilon>\Phi\left(x_{1}, x_{2}, \lambda_{2}\right),
$$

$$
f\left(x_{0}\right)-\varepsilon<f_{n}(x)=\psi\left(x_{1}, x_{2}, \lambda_{1}, \lambda_{2}\right)<f\left(x_{0}\right)+\varepsilon
$$

$f_{n}(x)$ tend, par suite, vers $f(x)$, si $n$ croît indéfiniment.

Ceci a lieu, en particulier, si $f(x)$ est' approximativement continue de deux côtés.

Si $f(x)$ est seulement à prépondérance de continuité du côté droit (ou de deux côtés), c.-à.-d. si les tonctions

$$
\underset{a}{\operatorname{mes}} E\left\{f(x)<f\left(x_{0}\right)+\varepsilon\right\}, \quad \operatorname{mes}_{a}^{x_{0}} E\{f(x)<f(x)-\varepsilon\}
$$

1) Si $f(x)$ est définie dans un intervalle fini; nous lui attribuerons, pour dé. finir $f_{n}(x)$, des valeurs quelconques finis (p. ex. le valeur 0 ) hors de cet intervallo. 
ont an points $x_{0}$ leurs nombres dérivés à droite $>\frac{1}{2}$, il n'est pas permis de choisir $\lambda_{1}, \lambda_{2}$ d'une facon quelconque, indépendant de $n$, mais il faut poser $f_{n}(x)=\psi\left(x, x+\frac{1}{n}, \lambda_{1}(n), \lambda_{2}(n)\right)$, où $\lambda_{1}(n), \lambda_{2}(n)$ tendent vers $\frac{1}{2}$, si $n$ croît indéfiniment.

La démonstration de la convergence de la suite $f_{\mathrm{n}}(x)$ se fait alors comme pour les fonctions approximativement continues.

5. Fin admettant la proposition démontrée par M. Denjoy 1$)$ que toute fonction approximativement continue, ou à prépondérance do continaité, prend dans un intervalle quelconque toute valeur entre son mininum et son maximum (dans cet intervalle) ${ }^{2}$ ), on peut former une autre suite de fonctions continues. En effet. dans ce eas, $y$ étant une valeur quelconque entre le ninimum et le maximum de $f^{\prime}(x)$ dans $\left(x_{1}, x_{2}\right)$, l'ensemble $E\left\{y-\varepsilon<f^{\prime}(x)<y+\varepsilon\right\}$ a une mesure positive. De lì résulte que $\varphi\left(x_{1}, x_{2}, \lambda\right)=\Phi\left(x_{1}, x_{2}, \lambda\right)$ pour toute valeur de $\lambda$, comprise entre 0 et 1 . Done, $\varphi=\Phi$ est une fonction continue do $x_{1}, x_{2}$ s).

On voit aisément que la suite de fonctions continues $f_{n}(x)=$ $=\varphi\left(x, x+\frac{1}{n} ; \lambda\right)$ tend vers $f(x)$ (en prenant resp. pour $\lambda$ un nombre quelconque compris entre 0 et 1 , ou le nombre $\lambda=\frac{1}{2}$ ).

Fnfin, pour les fonctions approximativement continues de deux côtés, ou seulement du côté droit, on peut encore procéder comme il suit. Soit d'ubord $f(x)$ bornée; $f(x)$ est la dérivée à droite de son intégrale besguienne indéfinie $\left.H^{\prime}(x)^{\star}\right)$, done la limite de la suite de fonctions continues $\frac{F\left(x+h_{n}\right)-F(x)}{h_{n}}$, où $h_{n}>0$ tend vers 0 avec $\frac{1}{n}$. (Il est à rémarquer que $\frac{F^{\prime}\left(x+h_{n}\right)-F(x)}{h}=\int_{0}^{1} \varphi(x, x+h, \lambda) d \lambda$, l'intégrule étant celle de Riemann. Cette égalité subsiste si $f$ est

1) Rull. Soc. Math. 1910. p. 180, p. 184 (en note).

1) Cetto proposition n'ost pas exacto pour dos fonctions approximativement

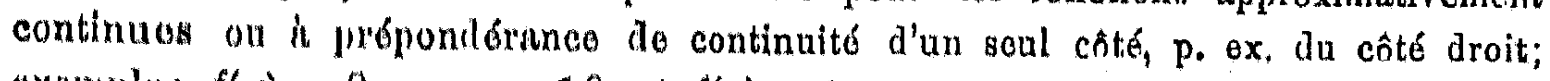
examplo: $f(x)=0$ pour $x<0$, et $f^{\prime}(x)=1$ pour $x \geqslant 0$ ). Elle lo devient, si l'on ajoutu fine $f^{\prime}(r)$ noitt toujours une des limites de $f(x)$, si $x$ tend vers $x_{0}$. en restant $\left\langle x_{10}\right.$; moyoununt cottis mupposition to raisonnement de $\$ 5$ s'applique aussi.

3) at antasi du $\lambda$. Do lì résalte qua: $\phi\left(x_{1}, x_{2}, \lambda\right)=\Phi\left(x_{1}, x_{2}, \lambda\right)=\lim \psi\left(x_{1}, x_{2}, \lambda_{1}, \lambda_{2}\right)$

9) Bull. Soo. Nath. 1915, p. 172. $\lambda_{1} \rightarrow \lambda$
$\lambda_{g} \rightarrow \lambda$ 
sommable, sans être bornée, mais alors $\int_{0}^{1}$ est une intégrale de Rie* mann généralisée). Si $f(x)$ n'est pas bornée, nous considérona la fonction bornée et approximativement continue $g(x)=\frac{f(x)}{1+|f(x)|}$ $g(x)$ est alors la limite de la suite des fonctions $g_{n}(x)=\frac{1}{n} \int_{*} g(x) d x$ et $f(x)$ celle de la suite $f_{n}(x)=\frac{g_{n}(x)}{1-\left|g_{n}(x)\right|}$.

\section{Les dérivées approximatives ot les nombres dórlvós prépondérants.}

6. Soit $F(x)$ une tonction continue de $x$. Il existe un nombre $\chi\left(x_{1}, x_{2}, \lambda\right)$ fini et un seul, satisfaisant aux conditions:

$$
\begin{aligned}
& \operatorname{mes}_{x_{1}} E\left\{\frac{F(x)-F\left(x_{1}\right)}{x-x_{1}}<\chi\left(x_{1}, x_{2}, \lambda\right)\right\} \leqslant \lambda\left(x_{2}-x_{2}\right) . \\
& \operatorname{mes}_{x_{1}}^{x_{1}} E\left\{\frac{F(x)-F\left(x_{1}\right)}{x-x_{1}} \leqslant \chi\left(x_{1}, x_{2}, \lambda\right)\right\} \geqslant \lambda\left(x_{1}-x_{1}\right) .
\end{aligned}
$$

$\chi\left(x_{1}, x_{2}, \lambda\right)$ n'est pas autre chose que le nombre $p\left(x_{1}, x_{2}, \lambda\right)$ $=\Phi\left(x_{1}, x_{2}, \lambda\right)$ calculé pour la fonction $\frac{F(x)-F\left(x_{1}\right)}{x-x_{1}}$, continue pour $x_{1}<x \leqslant x_{2}$.

On vérifie facilement que la fonction

$$
\operatorname{mes}_{x_{1}}^{x_{3}} E\left\{\frac{F(x)-F\left(x_{1}\right)}{x-x_{1}}<y\right\}
$$

$y$ étant un nombre quelconque, varie d'une façon continue arec $x_{1}$ et $x_{2}$. On en déduit que $\chi\left(x_{1}, x_{2}, \lambda\right)$ est une fonction continue de $x_{1}, x_{2}$.

Done, les fonctions $f_{n}(x)=\chi\left(x, x+\frac{1}{n}, \lambda\right)$ constituont uno suito de fonetions continues.

7. Comme au $\& 4$, on démontre que $f_{u}^{\prime}(x)$ tend vers une limite $f(x)$,

$1^{0}$ Si $F(x)$ possède une dérivée approximative $f\left(x_{0}\right)$ pour los deux côtés, ou seulẹment pour le côté droit, c.-à.-d. si la fonotion 
$\operatorname{mes}_{a}^{\infty}\left\{\left|\frac{\left.F(x)-x_{0}\right)}{x-x_{0}}-f(x)\right|<\varepsilon\right\}$ possède au point $x_{0}$ une dérivée, ou seulement une dérivée a droite, égale à un, $\lambda$ peut etre choisi un nombre quelconque compris entre 0 et 1 .

$2^{\circ}$ Si $F^{\prime}(x)$ possède un dérivé prépondérant bilatéral, ou seulement à droite, o.mà.- $d$. si la fonction

$$
\operatorname{mes}_{n} E^{\prime}\left\{\left|\frac{H^{\prime}(x)-F^{\prime}\left(x_{0}\right)}{x-x_{0}}-f\left(x_{0}\right)\right|<\varepsilon\right\}
$$

possède au point $x_{0}$ ses dérivés extrêmes inférieurs, ou seulement son numbre dérivé infériour a droite $>{ }^{1}$ ), dans ce cas $\lambda$ doit Atre egal a 1 .

1) Il auflat mêmo do supposer quo

$$
\operatorname{men}_{-1} x\left\{\left|\frac{F^{\prime}(x)-F^{\prime}\left(x_{0}\right)}{x-x_{0}}-f(x)\right|<\varepsilon\right\}>\frac{h}{2}
$$

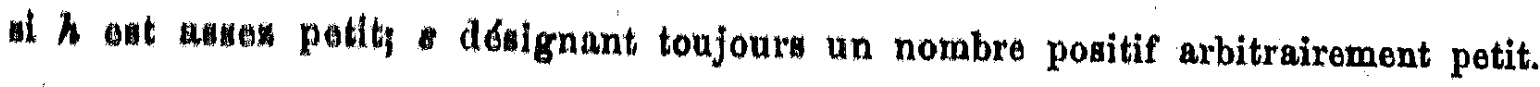

